\title{
Avaliação da presença de cuidador familiar de idosos com déficits cognitivo e funcional residentes em Belo Horizonte-MG
}

\author{
Evaluation of the presence of family caregivers of elderly with cognitive and functional deficits \\ living in Belo Horizonte-MG, Brazil
}

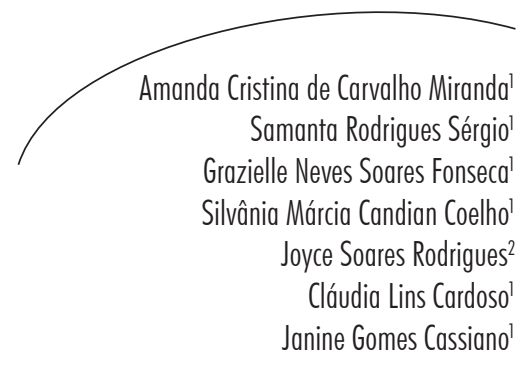

Resumo

Objetivo: Detectar a população idosa com declínio cognitivo e funcional moradora da região abrangida por uma unidade básica de saúde (UBS) em Belo Horizonte-MG, descrever seu perfil sociodemográfico e avaliar a presença de cuidador familiar. Método: Estudo transversal a partir de pesquisa do Programa de Educação pelo Trabalho em Saúde (PET-Saúde), na Linha de Cuidados de Saúde do Idoso. Do total da amostra, foram selecionados para análise idosos com escore do Miniexame do Estado Mental (MEEM) menor que 18, totalizando 91 indivíduos. As variáveis sociodemográficas analisadas foram idade, gênero, renda, escolaridade, morar sozinho e ter cuidador familiar. Também foi analisada a força de preensão manual. Resultados: 25\% dos idosos entrevistados apresentaram MEEM menor que 18. Destes, $80 \%$ eram mulheres; as idades variaram entre 60 e 96 anos; $12 \%$ moravam sozinhos; 85\% não tinham cuidador familiar. No geral, apresentaram baixos graus de instrução e renda; 52,31\% dos que foram submetidos à medida de força de preensão manual podem ser considerados préfrágeis. Conclusão: A maioria dos idosos deste estudo não possui cuidador familiar, além disso, há idosos com déficit cognitivo e funcional morando sozinhos.

\section{Abstract}

Objective: Detect elderly with cognitive and functional decline living in the area covered by a basic health unit (BHU) in Belo Horizonte-MG, Brazil, describe their profile and assess the presence of family caregivers. Method: Cross-sectional study from research of Programa de Educação pelo Trabalho em Saúde (PET-Saúde), in the área of Elderly Health Care. From the whole sample, were selected for analysis elderly individuals with Mini-Mental State Examination (MMSE) less than 18, totaling 91 individuals. We analyzed age, gender, income, education, grip strength, living alone and having family caregiver. Results: $25 \%$ of the subjects interviewed had MMSE less than 18. Among these, $80 \%$ were women, ages ranged between 60 and 96 years, $12 \%$ lived alone, $85 \%$

Palavras-chave: Idoso. Idoso Fragilizado. Vulnerabilidade em Saúde. Cuidadores. Família.

Key words: Elderly. Frail Elderly. Health Vulnerability. Caregivers. Family.

\footnotetext{
Universidade Federal de Minas Gerais. Programa de Educação pelo Trabalho em Saúde. Belo Horizonte, MG, Brasil.

2 Assessoria de Gestão Estratégica e Inovação/Secretaria de Estado de Educação de Minas Gerais/ Governo de Minas Gerais. Belo Horizonte, MG, Brasil.
} 
had no family caregiver. Overall, they had low levels of education and income; $52.31 \%$ of those who underwent handgrip strength measurement can be considered pre-frail. Conclusion: Most elderly in this study do not have family caregivers, in addition, there are elderly with cognitive and functional deficits living alone.

\section{INTRODUÇÃo}

O aumento da longevidade dos brasileiros e a incidência das doenças crônicas e/ou incapacitantes explicam o crescimento do número de idosos que necessitam de algum tipo de ajuda para realizar tarefas do cotidiano, como por exemplo, preparar refeições, fazer faxina ou compras. Cerca de $40 \%$ das pessoas com 65 anos de idade ou mais precisam de ajuda para essas tarefas do dia a dia, enquanto uma parcela de $10 \%$ precisa de auxílio nas tarefas básicas, como se vestir, tomar banho, comer e se levantar de uma cadeira. ${ }^{1,2}$

A falta de um cuidador para um idoso portador de morbidade acompanhada de declínio funcional e grau de dependência elevada é classificada como vulnerabilidade social..$^{3-5} \mathrm{O}$ conceito de vulnerabilidade é complexo e abrange várias dimensões, como a individual, relacionada ao comportamento e ao conhecimento dos riscos e agravos; programática, associada à relação deficitária do indivíduo com os serviços de saúde e, por fim, social, que considera o perfil populacional da área de abrangência, os níveis culturais de acesso à informação e os gastos com saúde. De modo sucinto, a vulnerabilidade pode ser definida como a capacidade de um indivíduo sofrer dano quando submetido a determinado estímulo. ${ }^{3-5}$ Isso torna relevante a identificação de casos de idosos com baixa cognição sem a presença de um cuidador.

Além disso, o conceito de fragilidade se refere a uma síndrome clínica na qual há redução da reserva de estressores e resistência diminuída a eles, como consequência do declínio dos sistemas fisiológicos. ${ }^{6}$ Em Belo Horizonte-MG, 13,27\% da população de pessoas com idade igual ou superior a 65 anos são frágeis, de acordo com os critérios de Fried et al., ${ }^{6}$ enquanto 59,29\% estão na préfragilidade. ${ }^{7}$ Fried et al. ${ }^{6}$ estabeleceram fenótipos para serem marcadores dessa condição de fragilidade, tais como: declínio de peso corporal não intencional (perda de mais de $4,5 \mathrm{~kg}$ ou mais de $5 \%$ do peso corporal em um ano), fraqueza (força de preensão manual abaixo de $20 \%$ do valor normal para o gênero e massa corporal correspondente), baixa resistência (exaustão avaliada por autorrelato de fadiga indicado por duas questões da Center for Epidemiological StudiesDepression scale - CES-D scale), lentidão (medida pela velocidade da marcha em segundos) e baixo nível de atividade física, baseada na pontuação de perda de quilocalorias gastas semanalmente.

O idoso que apresenta três ou mais desses fenótipos é classificado como frágil, enquanto que a presença de um ou dois desses fenótipos, como pré-frágil. O declínio acumulado de múltiplos sistemas fisiológicos o torna suscetível a eventos adversos como doenças, variações ambientais e acidentes, podendo culminar em declínio funcional, quedas, hospitalização e até mesmo falecimento do idoso. ${ }^{6,8,9}$

De acordo com o Estatuto do Idoso $\left(\operatorname{art} .3^{\circ}\right),{ }^{10}$ a obrigação de cuidar do bem-estar da pessoa idosa e assegurar seus direitos é da família, da comunidade, da sociedade e do poder público. Para atender a essa lei, foi instituída em 19 de outubro de 2006, a Política Nacional de Saúde da Pessoa Idosa, por meio da Portaria n ${ }^{\circ}$ 2.528/ GM, cujo objetivo é garantir atenção adequada e digna para a população brasileira idosa, com medidas individuais e coletivas em todos os níveis de atenção à saúde. Algumas de suas estratégias incluem capacitação de profissionais da saúde para o cuidado com o idoso, fomento à pesquisa na área do envelhecimento e programas de acesso do idoso ao sistema de saúde. ${ }^{11}$ 
A realidade, no entanto, mostra que a maior parte da responsabilidade com o idoso ainda recai sobre a família, normalmente sobre um membro do gênero feminino, que se torna cuidador informal do parente idoso e que não recebe orientações, nem possui uma estrutura eficaz para prestar cuidados efetivos ao idoso. . $^{10,12,13}$

Esse cuidador é um membro familiar que, voluntariamente, presta cuidado ao idoso, auxiliando em tarefas como alimentação, locomoção, higiene pessoal, medicação de rotina, entre outros, contribuindo, ainda que parcialmente, para a recuperação e qualidade de vida do idoso. . $^{12,14,15}$

Diante do exposto busca-se, neste trabalho, detectar a população idosa com declínio cognitivo e funcional moradora da região abrangida por uma unidade básica de saúde (UBS) em Belo Horizonte-MG, bem como descrever seu perfil sociodemográfico e avaliar a capacidade funcional e presença de cuidador familiar.

\section{MÉTODOS}

Trata-se de estudo transversal que utilizou dados obtidos por meio de entrevistas realizadas no projeto de pesquisa Avaliação da situação de saúde de idosos residentes na área de abrangência de três Unidades Básicas de Saúde do município de Belo Horizonte, desenvolvido pelo Programa de Educação pelo Trabalho em Saúde (PET-Saúde), na Linha de Cuidado de Saúde do Idoso. A pesquisa original tinha como um de seus objetivos avaliar de forma global a situação de saúde do idoso em áreas atendidas pelo Programa de Saúde da Família. Entretanto, cumpre informar que os resultados aqui apresentados referem-se apenas àqueles relativos ao déficit cognitivo e funcional encontrados na amostra pesquisada de uma das UBSs.

A coleta de dados ocorreu entre dezembro de 2011 e março de 2012. Nas áreas de abrangência de cada Equipe de Saúde da Família (ESF), foi selecionado aleatoriamente um número de setores censitários (SC) determinado de acordo com a proporcionalidade do número de $\mathrm{SC}$ de cada UBS, garantindo distribuição espacial homogênea. A determinação do número de SC total selecionado foi feita com base em cálculo amostral aleatório, considerando toda a população idosa da área de estudo. A seleção dos SCs se deu por amostragem aleatória simples, em uma tabela de números aleatórios, adotando uma lista de SC para cada ESF com numerações independentes.

Os critérios de inclusão foram idosos de ambos os sexos, residentes nos SCs selecionados, com idade igual ou maior que 60 anos, no momento da entrevista e que aceitassem assinar o Termo de Consentimento Livre e Esclarecido (TCLE).

Foram utilizados como critérios de exclusão: pessoas com idade compatível ao critério de inclusão, mas não residentes de forma definitiva na casa visitada; pessoas sem condições de responder de forma satisfatória ao questionamento sociodemográfico do protocolo de coleta de dados; falta de condições de resposta a ser aceita pelo entrevistador em função da declaração de familiar ou de cuidador, que informasse a impossibilidade do idoso, ou atestada diretamente no início da entrevista, por meio de avaliação, no ato, da qualidade e segurança das respostas oferecidas às questões iniciais referidas (esses idosos foram computados na população amostral e classificados como impossibilitados de responder); idosos cuja avaliação pelo instrumento MEEM obtiveram escore igual ou inferior a 13; após informação da existência de idoso na residência visitada, ausência do mesmo no momento e em mais duas tentativas de entrevistas em visitas subsequentes; recusa explícita do idoso ou familiar responsável em participar da pesquisa; condições físicas que, no momento da visita, levassem os entrevistadores, familiares ou cuidadores a considerar a entrevista como um ato desconfortável para o idoso.

A coleta foi realizada por meio de entrevista estruturada (protocolo de coleta). Os entrevistadores foram treinados por professores tutores e profissionais de saúde de diferentes formações, preceptores desses alunos em suas atividades nas respectivas UBS. Além disso, 
todas as definições necessárias ao preenchimento do Protocolo foram estabelecidas em manual. Os entrevistadores eram todos bolsistas e voluntários selecionados para o Programa de Educação no Trabalho em Saúde (PET-Saúde) da Universidade Federal de Minas Gerais (UFMG), em parceria com a Secretaria Municipal de Saúde de Belo Horizonte. As entrevistas foram realizadas sempre em dupla, estando os entrevistadores acompanhados pelos agentes comunitários de saúde da UBS, perfazendo 359 entrevistas. Os dados foram digitados em um banco de dados e tabulados em software de análise estatística.

Os dados clínicos e funcionais foram obtidos com base em diversos instrumentos, incluindo aqueles que avaliam a capacidade física e cognitiva, uma vez que a independência compreende a integridade dos dois fatores. ${ }^{16}$ Entretanto, o presente trabalho abordou somente o Miniexame do Estado Mental (MEEM) e a Força de Preensão Manual, validados para aplicação na língua portuguesa.

O MEEM é utilizado para rastreamento de déficit cognitivo de pessoas idosas e é composto por questões que abrangem orientação temporal e espacial, memória imediata e de evocação, praxia, cálculo e habilidades de linguagem, visão e espaço. Seu escore varia de 0 a 30 pontos, que estão associados, respectivamente, a maior declínio cognitivo e a maior capacidade cognitiva presumida. ${ }^{17,18}$ Entretanto, não existe na literatura consenso acerca do ponto de corte para detecção de déficit cognitivo, pois este é influenciado por muitos fatores, dentre os quais, a escolaridade.

Bertolucci et al. $^{19}$ encontraram, para o ensino fundamental, um corte de 18 pontos com sensibilidade de $75,6 \%$ e especificidade de $96,6 \%$. Brucki et al. ${ }^{20}$ definiram o ponto de corte como 20 para analfabetos e 25 para indivíduos com escolaridade superior a um ano. Por sua vez, o trabalho de Lourenço \& Veras ${ }^{21}$ revelou um corte de 18/19 para analfabetos (sensibilidade: $73,5 \%$ e especificidade: $73,9 \%$ ) e de $24 / 25$ para aqueles com instrução escolar (sensibilidade: $75 \%$ e especificidade: 69,7\%). O escore inferior a 18 pontos foi escolhido como ponto de corte para este estudo, pois evita muitos falsos positivos. Em outras palavras, o valor 18 apresenta menor erro ao classificar os idosos quanto ao possível declínio cognitivo. ${ }^{20,21}$

A força de preensão manual foi medida pelo dinamômetro JAMAR, considerado de boa confiabilidade, validade e de alta precisão. ${ }^{22}$ Para realizar o teste, o paciente permaneceu na posição sentada, com a coluna ereta, pernas fletidas formando um ângulo de $90^{\circ} \mathrm{com}$ as coxas e seu cotovelo (o da mão dominante) fletido a $90^{\circ}$, com o antebraço na posição neutra. Para análise dos dados, foram utilizados os valores de referência estabelecidos por Bohannon et al. ${ }^{23}$ que se associam ao gênero e faixa etária e podem ser divididos em regular, bom e excelente (tabelas 1 e 2).

Tabela 1. Valores de referência de preensão manual (kg) esquerda e direita para homens (95\% CI). Belo Horizonte-MG, 2012. ${ }^{23}$

\begin{tabular}{lcccccc}
\hline & \multicolumn{3}{c}{ Mão esquerda } & \multicolumn{3}{c}{ Mão direita } \\
\hline Idade(anos) & Regular & Bom & Excelente & Regular & Bom & Excelente \\
\hline $60-64$ & $<33,4$ & $33,4-44,0$ & $>51,1$ & $<36,8$ & $36,8-46,7$ & $>46,7$ \\
$65-69$ & $<32,0$ & $32,0-44,4$ & $>44,4$ & $<35,4$ & $35,4-47,9$ & $>47,9$ \\
$70-74$ & $<30,3$ & $30,3-42,1$ & $>42,1$ & $<32,0$ & $32,0-44,5$ & $>44,5$ \\
$75+$ & $<24,8$ & $24,8-34,7$ & $>34,7$ & $<12,7$ & $12,7-31,0$ & $>31,0$ \\
\hline
\end{tabular}


Tabela 2. Valores de referência de preensão manual $(\mathrm{kg})$ esquerda e direita para mulheres $(95 \% \mathrm{CI})$. Belo Horizonte-MG, 2012. ${ }^{23}$

\begin{tabular}{ccccccc}
\hline \multicolumn{5}{c}{ Mão esquerda } & & Mão direita \\
\hline Idade(anos) & Regular & Bom & Excelente & Regular & Bom & Excelente \\
\hline $60-64$ & $<18,6$ & $18,6-27,3$ & $>27,3$ & $<22,2$ & $22,2-29,6$ & $>29,6$ \\
$65-69$ & $<19,6$ & $19,6-26,2$ & $>26,2$ & $<22,5$ & $22,5-28,8$ & $>28,8$ \\
$70-74$ & $<19,1$ & $19,1-25,8$ & $>25,8$ & $<20,7$ & $20,7-27,8$ & $>27,8$ \\
$75+$ & $<14,7$ & $14,7-18,1$ & $>18,1$ & $<19,9$ & $16,0-19,9$ & $>19,9$ \\
\hline
\end{tabular}

Neste estudo, foram selecionados 91 idosos, os quais obtiveram pontuação no MEEM menor que 18. Para esses idosos, foram analisadas as seguintes características sociodemográficas: idade, gênero, se mora sozinho, se possui cuidador familiar autorreferido, força de preensão manual, renda e escolaridade. As análises foram realizadas no programa Statistical Package for the Social Sciences (SPSS v. 15).

Em cumprimento aos critérios éticos, o presente estudo foi submetido e aprovado pelo Comitê de Ética em Pesquisa da Secretaria Municipal de Saúde de Belo Horizonte, com o protocolo número 410.203/09, desenvolvido em parceria com a Universidade Federal de Minas Gerais (UFMG) e a Secretaria Municipal de Saúde de Belo Horizonte. Antes do preenchimento do protocolo, os idosos e seus familiares (quando presentes) foram informados sobre os objetivos da pesquisa e, caso aceitassem participar, foram solicitados a assinar o TCLE.

\section{RESULTADOS}

A entrevista foi feita com 359 idosos, dentre os quais 91 apresentaram escore do MEEM inferior a 18 , correspondendo a $25,4 \%$ do total de idosos entrevistados. No grupo de idosos com MEEM menor que 18, 79,1\% eram do gênero feminino. Apresentaram idades entre 60 e 96 anos, com maior concentração, na sétima e oitava décadas de vida. Quanto à renda, 58,1\% recebiam entre 0,5 e dois salários mínimos; $40 \%$ entre dois e cinco salários e 1,8\%, entre cinco e dez. O valor do salário mínimo neste estudo se refere ao da época da entrevista.

Em relação à estrutura familiar, 12,1\% dos idosos com MEEM inferior a 18 moravam sozinhos e $84,6 \%$ moravam com pelo menos mais uma pessoa. Cerca de 3\% não responderam a essa questão. Além disso, aproximadamente $85 \%$ não apresentaram cuidador familiar - que assim se identificava -, e apenas 15\% o possuíam. A maioria desses cuidadores familiares era do sexo feminino e tinha, em média, 52 anos, sendo que $50 \%$ possuíam idade superior a 53 anos. A idade máxima encontrada entre os cuidadores foi de 81 anos.

A relação entre idade e resultado do MEEM pode ser observada a partir de uma correlação. A estimativa do teste de correlação de Spearman medida não paramétrica utilizada para mensurar a força da relação entre duas variáveis - retornou coeficiente de correlação negativo, igual a $-0,31$. Isto significa que, apesar de a correlação não ser tão forte, existe associação negativa e estatisticamente significativa entre a idade e o resultado do MEEM, indicando que quanto maior a idade do indivíduo, menor tende a ser o resultado do teste e, portanto, maior grau de declínio cognitivo (tabela 3). 
Tabela 3. Correlação de Spearman entre idade e resultado do MEEM. Belo Horizonte-MG, 2012.

\begin{tabular}{|c|c|c|c|c|}
\hline \multicolumn{5}{|c|}{ Correlations } \\
\hline & & & I.3 & II_TOTAL \\
\hline \multirow[t]{6}{*}{ Spearman's rho } & I. 3 & Correlation Coefficient & 1,000 & $-0,309$ \\
\hline & & Sig. (2-tailed) & - & 0,003 \\
\hline & & $\mathrm{n}$ & 91 & 91 \\
\hline & II_TOTAL & Correlation Coefficient & $-0,309$ & 1,000 \\
\hline & & Sig. (2-tailed) & 0,003 & - \\
\hline & & $\mathrm{n}$ & 91 & 91 \\
\hline
\end{tabular}

Cumpre destacar que os idosos com declínio cognitivo analisados apresentaram grau de instrução até o ensino fundamental completo. A maioria $(52 \%)$ cursou o ensino fundamental incompleto e a segunda maior parcela (44\%) relatou nunca ter ido à escola.

Não se observa grande diferença entre o resultado do MEEM e o grau de instrução do idoso. A diferença entre a média do MEEM dos idosos que nunca foram à escola e os que tinham ensino fundamental incompleto foi de 0,26 pontos. Em relação aos que apresentaram ensino fundamental completo, essa diferença foi um pouco maior - 1,33 pontos. Embora tais diferenças indiquem que quanto maior o grau de instrução do idoso, maior o resultado médio do MEEM, elas não foram estatisticamente significantes.
Outro ponto interessante a se destacar é que $22 \%$ dos idosos com MEEM inferior a 18 não tiveram capacidade de realizar o teste de força de preensão manual, e $50 \%$ dos que foram capazes apresentaram força de preensão manual menor ou igual a 19,33 kgf. Além disso, aproximadamente $20 \%$ destes idosos, que foram capazes de realizar o teste de força de preensão manual, podem ser considerados como pré-frágeis, pois revelaram força menor que $13 \mathrm{kgf}^{6}$

De acordo com as referências de força de preensão manual estabelecidas por Bohannon et al., ${ }^{23}$ que leva em consideração o sexo, a faixa etária e a mão dominante, cerca de $50 \%$ desses idosos apresentaram força de preensão manual regular. Isto indica, mais uma vez, pré-fragilidade de praticamente a metade desses indivíduos (tabela 4). 
Tabela 4. Situação dos idosos quanto à força de preensão manual segundo as referências estabelecidas por Bohannon et al. ${ }^{23}$ Belo Horizonte-MG, 2012.

\begin{tabular}{lccc}
\hline Situação & Idosos & $(\%)$ & $(\%)$ válidos \\
\hline Bom & 19 & 20,9 & 29,23 \\
Excelente & 12 & 13,2 & 18,46 \\
Regular & 34 & 37,4 & 52,31 \\
\hline Sem medidas & 26 & 28,6 & - \\
Total & 91 & 100,0 & 100,0 \\
\hline
\end{tabular}

\section{DISCUSSÃO}

Neste estudo, observou-se que a maioria dos cuidadores familiares eram mulheres com idade média de 52 anos, resultado condizente com outros estudos. Gaioli et al. ${ }^{24}$ encontraram valor de $83 \%$ de cuidadoras; $80 \%$ tinham faixa etária igual ou superior a 46 anos. No estudo de Gratão et al., ${ }^{25} 80 \%$ eram mulheres, com idade média de 52,3 anos. Esses dados mostram que o fato de o cuidador familiar ser também um idoso ou uma pessoa de meia idade, muitas vezes com patologias associadas, pode dificultar a prestação do cuidado, além de torná-lo fragilizado e vulnerável. Igualmente, a possibilidade de ser um único cuidador em tempo integral, sem revezamento ou a existência de dependências mútuas entre cuidador/idoso, pode se tornar um fator que dificulte a decisão de o parente do idoso fragilizado assumir-se como cuidador. ${ }^{12}$

Em relação à presença de cuidadores familiares, os resultados deste estudo divergiram de outros dados da literatura. Apenas 15\% dos idosos com déficit cognitivo e funcional apresentaram cuidador familiar. No estudo de Gratão et al., ${ }^{25}$ este valor foi de 69,2\%. No trabalho de Gaioli et al., ${ }^{24}$ foi feito estudo descritivo dos cuidadores de idosos, sendo que, destes, 94\% eram cuidadores familiares.

A identificação de casos de idosos com baixa cognição sem a presença de um cuidador é de grande relevância, pois a falta de um cuidador para o idoso portador de declínio cognitivo, acompanhado de declínio funcional e grau de dependência elevada, é classificada como vulnerabilidade, o que implica necessidade de atendimentos especiais. ${ }^{6,7}$ No entanto, não se pode afirmar que os $85 \%$ dos idosos apontados como não possuidores de cuidador familiar não apresentem outro tipo de cuidador, pois uma das limitações do estudo se refere à não identificação da presença de um cuidador remunerado.

Esses resultados revelam a pré-fragilidade e a vulnerabilidade dosidosos analisados, uma vez que não há uma pessoa auxiliando-os a desempenhar tarefas rotineiras, ficando os mesmos responsáveis por cuidar de sua alimentação e higiene, administrar seus medicamentos e, de modo geral, gerenciar a própria vida. Para o idoso com déficit cognitivo e sem o auxílio por parte de outrem, muitas dessas tarefas podem apresentar risco ou ser realizadas de modo inadequado. Algo que pode ilustrar essa condição é o fato de pacientes idosos usarem, em média, de três a quatro tipos de medicamentos em diferentes horários durante o dia, aumentando a chance de erro na autoadministração da medicação. ${ }^{26}$

Por algum motivo, os familiares não podem, não querem ter a responsabilidade de cuidar ou não percebem a necessidade de cuidados ao idoso. Possíveis causas para esta constatação podem ser citadas: confusão, pela família, de sintomas demenciais com as manifestações do processo fisiológico de envelhecimento; o fato de 
o familiar trabalhar fora de casa e não ter tempo para se dedicar ao idoso e de não possuir recursos financeiros suficientes para contratar um cuidador formal ou parar de trabalhar; a presença de um problema de saúde no familiar que o incapacite para o cuidado ao idoso; a necessidade de o familiar cuidar de outro membro da família; a história e a dinâmica da família; os sacrifícios da atual vida social; o aumento da sobrecarga e problemas emocionais e físicos, tais como cansaço, estresse, irritabilidade e problemas de sono. ${ }^{12,18,27}$ Estas causas apontam a necessidade de ações que tenham o cuidador como público-alvo principal, uma vez que é de suma importância que os cuidadores sejam capacitados, para poder prestar auxílio com qualidade. ${ }^{28}$

Ademais, a maioria dos idosos (52,3\% daqueles que realizaram o teste) apresentou força de preensão manual regular. Pode-se constatar que boa parcela desses idosos possuem pelo menos um fenótipo de fragilidade, o que pode elevar, por exemplo, as chances de quedas e internações. Este resultado corrobora a necessidade de atenção especial aos idosos pré-frágeis, que moram sozinhos e com poucos recursos para contratar um cuidador formal. Para esses casos, sugere-se, por exemplo, um profissional contratado pelo próprio Estado. Em Belo Horizonte, essa medida tornou-se viável em 2011, por meio do Programa Maior Cuidado, que consiste na contratação de cuidador de idoso capacitado para idosos semidependentes e dependentes em situação de vulnerabilidade social. Além disso, o programa também conta com o projeto Domić́lio Seguro para a Pessoa Idosa, o qual fornece um kit banheiro, com o objetivo de garantir segurança e autonomia para os idosos em suas moradias. ${ }^{29}$

Uma das limitações encontradas neste estudo foi a falta de dados suficientes para detectar a quantidade de idosos frágeis, uma vez que são necessários três ou mais fenótipos, supracitados, para classificar um idoso como frágil. No presente estudo, considerou-se apenas um dos critérios de fragilidade; portanto, é possível afirmar apenas se há idosos pré-frágeis na amostra estudada. Além disso, não há informações sobre a existência de outro tipo de cuidador do idoso, como, por exemplo, o cuidador formal, embora seja pouco provável encontrá-lo na população analisada, uma vez que sua renda familiar é baixa.

\section{CONCLUSÃO}

Diante do exposto, observa-se a necessidade de ampliar estratégias que visem à conscientização da família do idoso dependente para a importância do cuidado familiar.

Medidas como qualificação dos cuidadores, sensibilização da família para maior envolvimento, esclarecimentos sobre as questões que acompanham o envelhecimento e formação de grupos de convivência podem ser alternativas interessantes, uma vez que não demandam custo significativo para os programas públicos. Em suma, cabe ao profissional de saúde e aos formuladores de políticas públicas destinadas aos idosos valorizar a rede de suporte ao idoso dependente.

Nesse sentido, éimportante a criação de espaços de discussão com dinâmicas educativas, grupos de convivência, bem como parcerias com outros setores que proporcionem ao idoso e ao cuidador práticas saudáveis de atividades, melhorando a qualidade de vida e prevenindo agravos. Esta rede tem papel fundamental ao atuar como alicerce do processo de cuidar com qualidade de uma parcela significativa de indivíduos que gozam de direitos de cidadania que lhes são específicos.

Vale ressaltar que este trabalho apresenta pretensões descritivas e que pode evoluir para análises mais sofisticadas em estudos vindouros. 


\section{REFERÊNCIAS}

1. Karsch UM. Dependent seniors: families and caregivers. Cad Saúde Pública 2003;9(3):861-6.

2. Santos CCC, Pedrosa R, Costa FA, Mendonça KMPP, Holanda GM. Análise da função cognitiva e capacidade funcional em idosos Hipertensos. Rev Bras Geriatr Gerontol 2011;14(2):241-50.

3. Bertolozzi MR, Nichiata LYI, Takahashi RF, Ciosak SI, Paula Hino, Do Val LF, et al. Os conceitos de vulnerabilidade e adesão na saúde coletiva. Rev Esc Enferm USP 2009;43(Esp 2):1326-30.

4. Lima LCV, Bueno CMLB. Envelhecimento e gênero: a vulnerabilidade de idosas no Brasil. Rev Saúde Pesq 2009;2(2):273-80.

5. Paz AA, Santos BRL, Eidt OR. Vulnerabilidade e envelhecimento no contexto da saúde. Acta Paul Enferm 2006;19(3):338-42.

6. Fried LP, Tangen C, Walston J, Newman A, Hirsch $\mathrm{C}$, Gottdiener J, et al. Frailty in older adults: evidence for a phenotype. J Gerontol Ser A, Biol Sci Med Sci 2001;56A(3):p. M146-156.

7. Santos EGS. Perfil de fragilidade em idosos comunitários de Belo Horizonte: um estudo transversal [dissertação]. Minas Gerais: Universidade Federal de Minas Gerais, Escola de Educação Física, Fisioterapia e Terapia Ocupacional; 2008.

8. Teixeira INAO. Percepções de profissionais de saúde sobre duas definições de fragilidade no idoso. Ciênc Saúde Coletiva 2008;13(4):1181-8.

9. Duarte YAO. Indicadores de fragilidade em pessoas idosas visando o estabelecimento de medidas preventivas. BIS, Bol Inst Saúde 2009;(47):49-52.

10. Brasil. Lei n. 10.741 , de $1^{\circ}$ de outubro de 2003. Dispõe sobre o Estatuto do Idoso e dá providências. Portal do Planalto. 2003. Disponível em: http://www.planalto.gov.br/ccivil_03/ leis/2003/L10.741.htm.

11. Brasil. Portaria n 2.528 , de 19 de outubro de 2006. Aprova a Política nacional de saúde da pessoa idosa. 2006. Disponível em: http://dtr2001.saude.gov.br/ sas/PORTARIAS/Port2006/GM/GM-2528.htm.

12. Born T, organizadora. Cuidar melhor e evitar violência: manual do cuidador da pessoa idosa. Brasília: Secretaria Especial dos Direitos Humanos, Subsecretaria de Promoção e Defesa dos Direitos Humanos; 2008.
13. Souza WGA, Pacheco WNS, Martins JJ, Barra DCC, Nascimento ERP. Educação em saúde para leigos no cuidado ao idoso no contexto domiciliar. ACM Arq Catarin Med 2006;35(4):56-63.

14. Brasil. Ministério da Saúde. Secretaria de Atenção à Saúde. Departamento de Atenção Básica. Envelhecimento e saúde da pessoa idosa. Brasília, DF: Ministério da Saúde; 2006. Cadernos de Atenção Básica, no 19. Série A. Normas e Manuais Técnicos.

15. Ministério da Saúde. Secretaria de Atenção à Saúde. Secretaria de Gestão do Trabalho e da Educação na Saúde. Guia Prático do Cuidador. Brasília, DF: Ministério da Saúde; 2008. Série A. Normas e Manuais Técnicos.

16. Macêdo AML, Cerchiari EAN, Alvarenga MRM, Faccenda O, Oliveira MAC. Avaliação funcional de idosos com déficit cognitivo. Acta Paul Enferm 2012;25(3):358-63.

17. Chaves MLF. Testes de avaliação cognitiva: MiniExame do Estado Mental [Internet]. São Paulo: Academia Brasileira de Neurologia; [2008?] [acesso em 28 Fev 2012]; 30 p. Disponível em: http://www. cadastro.abneuro.org/site/arquivos_cont/8.pdf

18. Jones RN, Gallo JJ. Dimensions of the Mini-Mental State Examination among community dwelling older adults. Psychol Med 2000;30(3):605-18.

19. Bertolucci PH, Brucki SM, Campacci SR, Juliano Y. The Mini-Mental State Examination in a general population: impact of educational status. Arq Neuropsiquiatr 1994;52(1):1-7.

20. Brucki SMD, Nitrini R, Caramelli P, Bertolucci PHF, Okamoto IH. Sugestões para o uso do mini-exame do estado mental no Brasil. Arq Neuropsiquiatr 2003;61(3B):777-81.

21. Lourenço RA, Veras RP. Mini-Exame do Estado Mental: características psicométricas em idosos ambulatoriais. Rev Saúde Pública 2006;40(4):712-9.

22. Figueiredo IM, Sampaio RF, Mancini MC, Silva FCM, Souza MAP. Teste de força de preensão utilizando o dinamômetro Jamar. Acta Fisiátrica 2007;14(2): 104-10.

23. Bohannon RW, Peolsson A, Massy-Westropp N, Desrosiers J, Bear-Lehman J. Reference values for adult grip strength measured with a Jamar dynamometer: a descriptive meta-analysis. Physiotherapy 2006;92(1):11-5.

24. Gaioli CCLO. Cuidadores de idosos com doença 
de Alzheimer: variáveis sociodemográficas e da saúde associadas à resiliência [tese]. Ribeirão Preto: Universidade de São Paulo, Escola de Enfermagem de Ribeirão Preto; 2010.

25. Gratão ACM. Demanda do cuidador familiar com idoso demenciado [dissertação]. Ribeirão Preto: Universidade de São Paulo, Escola de Enfermagem de Ribeirão Preto; 2006.

26. Nunes LM, Portella MR. O idoso fragilizado no domicílio: a problemática encontrada na atenção básica à saúde. Bol Saúde 2003;17(2):109-21.

27. Pavarini SCI, Melo LC, Silva VM, Orlandi FS, Mendiondo MSZ, Filizola CLA, et al. Cuidando de idosos com Alzheimer: a vivência de cuidadores familiares. Rev Eletr Enf [Internet] 2008 [acesso em 28 fev 2012]; 10(3):580-90. Disponível em: http://www. fen.ufg.br/fen_revista/v10/n3/pdf/v10n3a04.pdf.

28. Moreira MDC, Pereira C. A importância do cuidador no contexto da saúde do idoso. Esc Anna Nery Rev Enferm [internet] 2007 [acesso em 28 fev 2012]; 11(3):520-5. Disponível em: http://www.scielo.br/ pdf/ean/v11n3/v11n3a19.pdf.

29. Prefeitura de Belo Horizonte. BH Metas e Resultados. Programa de atenção ao idoso [internet]. Belo Horizonte, MG: Prefeitura de Belo Horizonte; [Acesso em 14 de set de 2014]. Disponível em: https://bhmetaseresultados.pbh.gov.br/content/ programa-de-atendimento-ao-idoso. 